\title{
Synthesis, Molecular Docking and Biological Evaluation of Substituted Quinazolinones as Antibacterial Agents
}

\author{
ARUN MAHATO*1 ${ }^{*}$ BIRENDRA SHRIVASTAVA ${ }^{2}$ and NITHYA SHANTHI ${ }^{1}$ \\ ${ }^{1}$ Department of Pharmaceutical Sciences, Sardar Bhagwan Singh PG Institute of Biomedical \\ Sciences \& Research, Balawala, Dehradun, 248161, India \\ ${ }^{2}$ School of Pharmaceutical Sciences, Jaipur National University, Jagatpura, \\ Jaipur, 302025, India \\ arunmahato@gmail.com
}

Received 25 November 2014 / Accepted 16 December 2014

\begin{abstract}
A series of 7-chloro-2,3-disubstituted-4(3H)-quinazolinones (4a-j) were synthesized by the reaction of 3-amino-7-chloro-2-phenylquinazolin-4(3H)-one (3) with various amines in presence of formaldehyde. The starting material for the compounds was 2-amino-4-chloro benzoic acid. The structures of the compounds were characterized by IR, ${ }^{1} \mathrm{H}$ NMR, elemental analyses. Docking analysis of quinazolinone derivatives was done with DNA gyrase protein which was carried out by means of the AutoDockTools (ADT) v 1.5.4 and AutoDock v 4.2 programs. All compounds interacted with DNA gyrase protein. Preclinical evaluation of the compounds was ascertained by in silico toxicity, blood-brain barrier and drug like properties. All compounds were investigated for their antibacterial activity. Overall, compound (4b) showed better properties as a drug like candidate.
\end{abstract}

Keywords: Quinazolinone, DNA gyrase, Docking, In silico toxicity, Antibacterial

\section{Introduction}

Quinazolines and condensed quinazolines are found to possess potent anticancer, antihistaminic, CNS activities like analgesic and anti-inflammatory, sedative-hypnotic, anticonvulsant and antibacterial activities ${ }^{1-9}$. Antibiotic resistance in the community is a growing public health concern due to the continued emergence of multi drug resistant bacterial strains. During the past decades, the human population affected with life threatening infectious diseases caused by multidrug resistant Gram-positive and Gramnegative pathogen bacteria increased to an alarming level around the world. Due to this reason, it is imperative to design and develop new antibacterial or antifungal agents with novel chemical structure preferably having different modes of action rather than analogues of the existing ones ${ }^{10}$.

The 4-quinolones such as ciprofloxacin, ofloxacin, lomefloxacin, gatifloxacin, are established synthetic antibacterial agents as DNA gyrase inhibitors ${ }^{11}$. Substituted quinazolines 
were reported as isosters of quinolones to show a variety of antibacterial activities with DNA gyrase inhibition ${ }^{12,13}$. DNA gyrase, consisting of the subunits GyrA and GyrB, is a member of the type II family of topoisomerases that control the topological state of DNA in cells $^{14}$. DNA gyrase couples ATP hydrolysis by the GyrB subunit to supercoiling of DNA, which is required for maintenance of DNA topology during the replication process. It is an essential enzyme across bacterial species and inhibition results in disruption of DNA synthesis and, subsequently, cell death ${ }^{15}$.

Substituted quinazolines were reported as isosters of quinolones to show a variety of antibacterial activities with DNA gyrase inhibition ${ }^{16}$. 4(3H)-Quinazolinones with 3 -substitution has been reported to be associated with antimicrobial properties ${ }^{17,18}$. The 3 -substitution which was reported is various substituted phenyl ring moieties ${ }^{19}$, bridged phenyl rings $^{20}$ heterocyclic rings ${ }^{21}$ and aliphatic systems ${ }^{22}$. 2,3-Substituted-4(3H)quinazolinones were reported to possess antimicrobial properties ${ }^{23}$. Quinoline derivatives is a recent study showed that some 7-chloro quinoline derivatives exhibited a significant antibacterial activity ${ }^{24}$. These observation led to the conception that a new series of 2-phenyl-3-(substituted methylamino)-(3H)-quinazolin-4-ones along with incorporation of chlorine in aromatic ring of the quinazolinone nucleus at the 7-position by molecular hybridization. The design concept of these compounds explores the introduction of substituted amines (A) into the 7-chloro-quinazolinone nucleus (B) to obtain 3-(substituted methylamino)-(3H)-quinazolin-4-ones (C) (Figure 1). The possibility of an involvement of the DNA-gyrase enzyme in the activity of quinazolines was confirmed from docking studies.

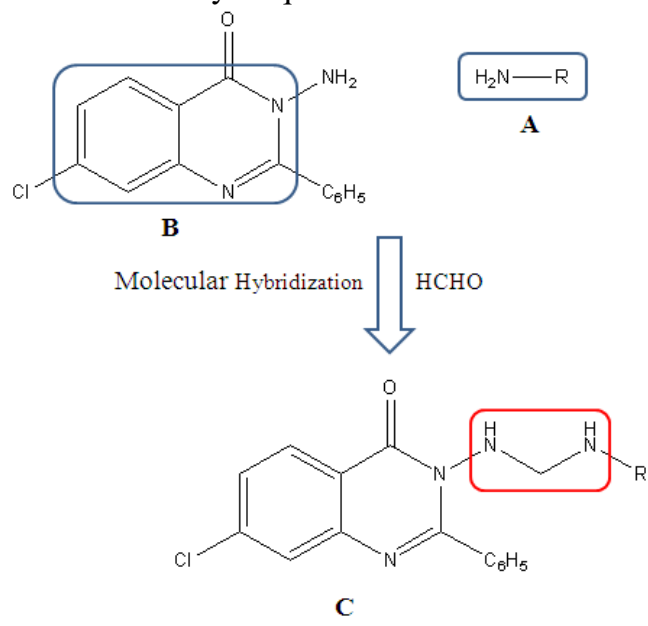

Figure 1. Design of Quinazolinone derivatives

\section{Experimental}

2-Amino-4-chloro benzoic acid was procured from Spectrochem, Mumbai. All other chemicals and reagents purchased from $\mathrm{CDH}$ and Rankem chemicals. Melting points of all the synthesized derivatives were determined by open-capillary tube method and values were uncorrected. IR spectra were recorded on Shimadzu FT/IR spectrometer in $\mathrm{cm}^{-1}$ values. ${ }^{1} \mathrm{H}$ NMR and spectra were recorded on a Bruker $400 \mathrm{MHz}$ NMR spectrometer using DMSO- $\mathrm{d}_{6}$ $/ \mathrm{CDCl}_{3}$ as the solvent.

The starting material 3-amino-7-chloro-2-phenylquinazolin-4(3H)-one (3) was synthesized according to the literature $\operatorname{method}^{25}$ (Figure 2). Compound (3) was used as precursor for 
further reaction with the purpose of synthesizing the several substituted quinazolinone at position 3. The final compounds (4a-4j) were synthesized from compound (3) by conjugation with various amines in presence of formalin.<smiles>Nc1cc(Cl)ccc1C(=O)O</smiles>

1

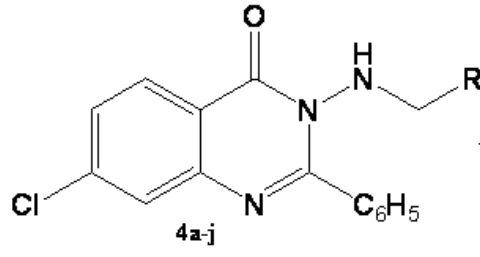

$4 a$ amino di ethyl

$4 \mathrm{~b}=$ amino dimethyl

$4 c=$ piperidinyl

$4 d=$ amino hyctroxyethyl

$4 e=$ amino benzyl

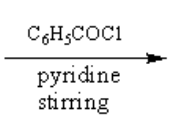<smiles>CCCCCCc1nc2cc(Cl)ccc2c(=O)o1</smiles>

$\mathrm{NH}_{2}-\mathrm{NH}_{2} \cdot \mathrm{H}_{2} \mathrm{O}$ reflux

Figure 2. Synthetic scheme for Quinazolinone derivatives

\section{Synthesis of 7-chloro-2-phenyl-3,1-benzoxazin-4-one (2)}

To a solution of 2-amino-4-chloro benzoic acid (1) $(0.1 \mathrm{~mol})$ dissolved in pyridine $(60 \mathrm{~mL})$, benzoyl chloride $(0.2 \mathrm{~mol})$ was added. The mixture was stirred for $30 \mathrm{~min}$ followed by treatment with $5 \% \mathrm{NaHCO}_{3}(15 \mathrm{~mL})$. The solid obtained was crystallized from ethanol, yield $=80 \%, \mathrm{mp} 168-170{ }^{\circ} \mathrm{C}$; IR $(\mathrm{KBr}) \mathrm{cm}^{-1}: 1780(\mathrm{C}=\mathrm{O}), 1680($ cyclic $\mathrm{C}=\mathrm{O}), 1620(\mathrm{C}=\mathrm{N})$. ${ }^{1} \mathrm{H}$ NMR (DMSO-d 6 , $\delta$ ppm): 8.32(s, 1H, 5-H), $7.94(\mathrm{~d}, 1 \mathrm{H}, 6-\mathrm{H}), 7.63(\mathrm{~d}, 1 \mathrm{H}, 8-\mathrm{H}), 6.84-$ $7.11\left(\mathrm{~m}, 5 \mathrm{H}, \mathrm{C}_{6} \mathrm{H}_{5}\right)$. Anal. calcd. for $\mathrm{C}_{14} \mathrm{H}_{8} \mathrm{ClNO}_{2}: \mathrm{C}, 65.26 ; \mathrm{H}, 3.13 ; \mathrm{N}, 5.44$. Found: $\mathrm{C}$, $65.16 ; \mathrm{H}, 3.23 ; \mathrm{N}, 5.32$.

\section{Synthesis of 3-amino-7-chloro-2-phenylquinazolin-4(3H)-one (3)}

A mixture of 7-chloro-2-phenyl-3,1-benzoxazin-4-one $(0.05 \mathrm{~mol})$ and hydrazine hydrate $(0.05 \mathrm{~mol})$ in ethanol was refluxed for $3 \mathrm{~h}$ and cooled. The separated solid was crystallized from ethanol, yield $=85 \%, \mathrm{mp} 235-238{ }^{\circ} \mathrm{C}$; IR $(\mathrm{KBr}) \mathrm{cm}^{-1}: 3300\left(\mathrm{NH}_{2}\right), 1700(\mathrm{C}=\mathrm{O}), 1620$ $(\mathrm{C}=\mathrm{N}) .{ }^{1} \mathrm{H}$ NMR (DMSO-d $\left.\mathrm{d}_{6}, \delta \mathrm{ppm}\right): 7.48-8.19(\mathrm{~m}, 8 \mathrm{H}, \mathrm{Ar}-\mathrm{H}), 5.67$ (s, 2H, 3-quinazolinone$\mathrm{NH}_{2}$ ). Anal. calcd. for $\mathrm{C}_{14} \mathrm{H}_{10} \mathrm{ClN}_{3} \mathrm{O}: \mathrm{C}, 68.39 ; \mathrm{H}, 3.71 ; \mathrm{N}, 15.47$. Found: C, 68.96; H, 3.53; $\mathrm{N}, 15.34$.

\section{General method of synthesis of 7-chloro-2,3-disubstituted-4(3H)-quinazolinones (4a-j)}

To a slurry of 3-amino-7-chloro-2-phenylquinazolin-4(3H)-one $(0.005 \mathrm{~mol})$ in dimethylformamide $(15 \mathrm{~mL})$ a mixture of formalin $(37-41 \%, 1 \mathrm{~mL})$ and various amines $(0.005 \mathrm{~mol})$ was added drop by drop with stirring. The reaction mixture was heated on a water bath for about $25 \mathrm{~min}$. After cooling, it was poured into ice water, the solid obtained was filtered, washed with water dried and recrystallized from ethanol. 
7-Chloro-3-((diethylamino)methylamino)-2-phenylquinazolin-4(3H)-one (4a)

Yield $=71 \%$, mp 166-167 ${ }^{\circ} \mathrm{C}$; IR $(\mathrm{KBr}) \mathrm{cm}^{-1}: 3280(\mathrm{NH}), 2860\left(\mathrm{CH}_{2}\right), 1700(\mathrm{C}=\mathrm{O}), 1620$ $(\mathrm{C}=\mathrm{N}) .{ }^{1} \mathrm{H}$ NMR $\left(\mathrm{CDCl}_{3}, \delta \mathrm{ppm}\right): 7.38-8.09(\mathrm{~m}, 8 \mathrm{H}, \mathrm{Ar}-\mathrm{H}), 3.67\left(\mathrm{~d}, 2 \mathrm{H},-\mathrm{CH}_{2}-\right), 2.31(\mathrm{q}, 4 \mathrm{H}$, $\left.\mathrm{CH}_{2}-\mathrm{CH}_{3}\right), 2.11(\mathrm{t}, 1 \mathrm{H}, \mathrm{NH}), 1.62\left(\mathrm{t}, 6 \mathrm{H}, \mathrm{CH}_{3}\right)$. Anal. calcd. for $\mathrm{C}_{19} \mathrm{H}_{21} \mathrm{ClN}_{4} \mathrm{O}: \mathrm{C}, 63.95 ; \mathrm{H}$, 5.93; N, 15.70. Found: C, 63.92; H, 5.13; N, 15.39.

\section{7-Chloro-3-((dimethylamino)methylamino)-2-phenylquinazolin-4(3H)-one (4b)}

Yield=65\%, mp 135-137 ${ }^{\circ} \mathrm{C}$; IR $(\mathrm{KBr}) \mathrm{cm}^{-1}: 3250(\mathrm{NH}), 2860\left(\mathrm{CH}_{2}\right), 1710(\mathrm{C}=\mathrm{O}), 1625$ $(\mathrm{C}=\mathrm{N}) .{ }^{1} \mathrm{H}$ NMR $\left(\mathrm{CDCl}_{3}, \delta \mathrm{ppm}\right): 6.83-7.9(\mathrm{~m}, 8 \mathrm{H}, \mathrm{Ar}-\mathrm{H}), 3.47\left(\mathrm{~d}, 2 \mathrm{H},-\mathrm{CH}_{2}-\right), 2.65(\mathrm{~s}, 6 \mathrm{H}$, $\left.\mathrm{N}\left(\mathrm{CH}_{3}\right)_{2}\right), 2.11(\mathrm{t}, 1 \mathrm{H}, \mathrm{NH})$. Anal. calcd. for $\mathrm{C}_{17} \mathrm{H}_{17} \mathrm{ClN}_{4} \mathrm{O}: \mathrm{C}, 62.10 ; \mathrm{H}, 5.21 ; \mathrm{N}, 17.04$. Found: C, 63.13; H, 5.31; N, 17.84.

\section{7-Chloro-2-phenyl-3-(piperidin-1-ylmethylamino)quinazolin-4(3H)-one (4c)}

Yield $=63 \%$, mp 145-147 ${ }^{\circ} \mathrm{C}$; IR $(\mathrm{KBr}) \mathrm{cm}^{-1}: 3350(\mathrm{NH}), 2880\left(\mathrm{CH}_{2}\right), 1650(\mathrm{C}=\mathrm{O}), 1605$ $(\mathrm{C}=\mathrm{N}), 1325(\mathrm{C}-\mathrm{N}) .{ }^{1} \mathrm{H}$ NMR $\left(\mathrm{CDCl}_{3}, \delta \mathrm{ppm}\right)$ : 6.93-7.97 (m, 8H, $\left.\mathrm{Ar}-\mathrm{H}\right), 3.47\left(\mathrm{~d}, 2 \mathrm{H},-\mathrm{CH}_{2}-\right)$, $2.25\left(\mathrm{t}, 4 \mathrm{H}, \mathrm{CH}_{2}\right), 2.19(\mathrm{t}, 1 \mathrm{H}, \mathrm{NH}), 1.52\left(\mathrm{~m}, 6 \mathrm{H}, \mathrm{CH}_{2}\right)$. Anal. calcd. for $\mathrm{C}_{20} \mathrm{H}_{21} \mathrm{ClN}_{4} \mathrm{O}: \mathrm{C}$, 65.12; H, 5.74; N, 15.19. Found: C, 65.92; H, 6.04; N, 15.89 .

\section{7-Chloro-3-((2-hydroxyethylamino)methylamino)-2-phenylquinazolin-4(3H)-one (4d)}

Yield $=73 \%$, mp 202-205 ${ }^{\circ} \mathrm{C}$; IR $(\mathrm{KBr}) \mathrm{cm}^{-1}: 3510(\mathrm{OH}), 3310(\mathrm{NH}), 2869\left(\mathrm{CH}_{2}\right), 1610(\mathrm{C}=\mathrm{O})$, $1635(\mathrm{C}=\mathrm{N}) .{ }^{1} \mathrm{H}$ NMR $\left(\mathrm{CDCl}_{3}, \delta \mathrm{ppm}\right): 6.73-8.27(\mathrm{~m}, 8 \mathrm{H}, \mathrm{Ar}-\mathrm{H}), 3.97\left(\mathrm{t}, 2 \mathrm{H}, \mathrm{CH}_{2}\right), 3.65(\mathrm{q}$, $\left.2 \mathrm{H}, \mathrm{CH}_{2}\right), 2.65\left(\mathrm{q}, 2 \mathrm{H}, \mathrm{CH}_{2}\right), 2.18(\mathrm{t}, 1 \mathrm{H}, \mathrm{OH}), 2.06(\mathrm{t}, 1 \mathrm{H}, \mathrm{NH}), 1.92(\mathrm{~m}, 1 \mathrm{H}, \mathrm{NH})$. Anal. calcd. for $\mathrm{C}_{17} \mathrm{H}_{17} \mathrm{ClN}_{4} \mathrm{O}_{2}$ : C, 59.22; H, 4.97; N, 16.25. Found: C, 60.02; H, 5.17; N, 16.65.

\section{3-((Benzylamino)methylamino)-7-chloro-2-phenylquinazolin-4(3H)-one (4e)}

Yield $=78 \%$, mp 212-214 ${ }^{\circ} \mathrm{C}$; IR (KBr) cm${ }^{-1}: 3385(\mathrm{NH}), 3039(\mathrm{CH}), 2890\left(\mathrm{CH}_{2}\right), 1610$ $(\mathrm{C}=\mathrm{O}), 1635(\mathrm{C}=\mathrm{N}) .{ }^{1} \mathrm{H}$ NMR $\left(\mathrm{CDCl}_{3}, \delta \mathrm{ppm}\right):$ 7.07-8.21 (m, 13H, $\left.\mathrm{Ar}-\mathrm{H}\right), 3.91\left(\mathrm{t}, 2 \mathrm{H}, \mathrm{CH}_{2}\right)$, $3.85\left(\mathrm{~d}, 2 \mathrm{H}, \mathrm{CH}_{2}\right), 2.06(\mathrm{t}, 1 \mathrm{H}, \mathrm{NH}) 1.96(\mathrm{~m}, 1 \mathrm{H}, \mathrm{NH})$. Anal. calcd. for $\mathrm{C}_{22} \mathrm{H}_{19} \mathrm{ClN}_{4} \mathrm{O}: \mathrm{C}$, 67.60; H, 4.90; N, 14.33. Found: C, 67.69; H, 4.89; N, 14.65.

\section{7-Chloro-3-((4-hydroxyphenylamino)methylamino)-2-phenylquinazolin-4(3H)-one (4f)}

Yield $=78 \%$, mp 212-214 ${ }^{\circ} \mathrm{C}$; IR (KBr) cm $\mathrm{cm}^{-1}: 3525(\mathrm{OH}), 3380(\mathrm{NH}), 3030(\mathrm{CH}), 2890$ $\left(\mathrm{CH}_{2}\right), 1610(\mathrm{C}=\mathrm{O}), 1639(\mathrm{C}=\mathrm{N}) .{ }^{1} \mathrm{H}$ NMR $\left(\mathrm{CDCl}_{3}, \delta \mathrm{ppm}\right): 6.27-8.21(\mathrm{~m}, 12 \mathrm{H}, \mathrm{Ar}-\mathrm{H}), 5.17$ $(\mathrm{s}, 1 \mathrm{H}, \mathrm{OH}), 4.41\left(\mathrm{t}, 2 \mathrm{H}, \mathrm{CH}_{2}\right), 4.06(\mathrm{t}, 1 \mathrm{H}, \mathrm{NH}), 2.1(\mathrm{t}, 1 \mathrm{H}, \mathrm{NH})$. Anal. calcd. for $\mathrm{C}_{21} \mathrm{H}_{17} \mathrm{ClN}_{4} \mathrm{O}_{2}$ : C, 64.21; H, 4.36; N, 14.26. Found: C, 65.01; H, 4.76; N, 14.15.

\section{7-Chloro-3-((diphenylamino)methylamino)-2-phenylquinazolin-4(3H)-one (4g)}

Yield $=58 \%, \mathrm{mp} 232-234{ }^{\circ} \mathrm{C}$; IR $(\mathrm{KBr}) \mathrm{cm}^{-1}: 3355(\mathrm{NH}), 3010(\mathrm{CH}), 2870\left(\mathrm{CH}_{2}\right), 1620$ $(\mathrm{C}=\mathrm{O}), 1640(\mathrm{C}=\mathrm{N}) .{ }^{1} \mathrm{H}$ NMR $\left(\mathrm{CDCl}_{3}, \delta \mathrm{ppm}\right): 6.47-8.05(\mathrm{~m}, 18 \mathrm{H}, \mathrm{Ar}-\mathrm{H}), 4.42(\mathrm{~d}, 2 \mathrm{H}$, $\mathrm{CH}_{2}$ ), 2.05 (t, $1 \mathrm{H}, \mathrm{NH}$ ). Anal. calcd. for $\mathrm{C}_{27} \mathrm{H}_{21} \mathrm{ClN}_{4} \mathrm{O}: \mathrm{C}, 71.60 ; \mathrm{H}, 4.67 ; \mathrm{N}, 12.37$. Found: C, 71.90; H, 4.14; N, 12.19 .

\section{7-Chloro-3-((4-methoxyphenylamino)methylamino)-2-phenylquinazolin-4(3H)-one (4h)}

Yield $=55 \%$, mp 197-198 ${ }^{\circ} \mathrm{C}$; IR (KBr) cm ${ }^{-1}: 3350(\mathrm{NH}), 3020(\mathrm{CH}), 2890\left(\mathrm{CH}_{2}\right), 1615$ $(\mathrm{C}=\mathrm{O}), 1640(\mathrm{C}=\mathrm{N}) .{ }^{1} \mathrm{H}$ NMR $\left(\mathrm{CDCl}_{3}, \delta \mathrm{ppm}\right): 6.37-7.93(\mathrm{~m}, 12 \mathrm{H}, \mathrm{Ar}-\mathrm{H}), 4.41\left(\mathrm{t}, 2 \mathrm{H}, \mathrm{CH}_{2}\right)$, $3.73\left(\mathrm{~s}, 3 \mathrm{H}, \mathrm{OCH}_{3}\right), 3.55(\mathrm{t}, 1 \mathrm{H}, \mathrm{NH}), 2.1(\mathrm{t}, 1 \mathrm{H}, \mathrm{NH})$. Anal. calcd. for $\mathrm{C}_{22} \mathrm{H}_{19} \mathrm{ClN}_{4} \mathrm{O}_{2}: \mathrm{C}$, 64.94; H, 4.71; N, 13.77. Found: C, 65.34; H, 4.21; N, 13.61. 
7-Chloro-3-((2-nitrophenylamino)methylamino)-2-phenylquinazolin-4(3H)-one (4i) Yield=59\%, mp 175-178 ${ }^{\circ} \mathrm{C}$; IR $(\mathrm{KBr}) \mathrm{cm}^{-1}: 3360(\mathrm{NH}), 3010(\mathrm{CH}), 2895\left(\mathrm{CH}_{2}\right), 1610(\mathrm{C}=\mathrm{O})$, $1630(\mathrm{C}=\mathrm{N}) .{ }^{1} \mathrm{H}$ NMR $\left(\mathrm{CDCl}_{3}, \delta \mathrm{ppm}\right): 6.73-7.97(\mathrm{~m}, 12 \mathrm{H}, \mathrm{Ar}-\mathrm{H}), 4.42\left(\mathrm{t}, 2 \mathrm{H}, \mathrm{CH}_{2}\right), 3.45(\mathrm{t}$, $1 \mathrm{H}, \mathrm{NH}), 2.1(\mathrm{t}, 1 \mathrm{H}, \mathrm{NH})$. Anal. calcd. for $\mathrm{C}_{21} \mathrm{H}_{16} \mathrm{ClN}_{5} \mathrm{O}_{3}: \mathrm{C}, 59.79 ; \mathrm{H}, 3.82 ; \mathrm{N}, 16.60$. Found: C, 59.98; H, 3.32; N, 16.75.

\section{7-Chloro-2-phenyl-3-((phenylamino)methylamino $)$ quinazolin-4(3H)-one (4j)}

Yield $=79 \%$, mp 215-218 ${ }^{\circ} \mathrm{C}$; IR $(\mathrm{KBr}) \mathrm{cm}^{-1}: 3330(\mathrm{NH}), 3010(\mathrm{CH}), 2890\left(\mathrm{CH}_{2}\right), 1610$ $(\mathrm{C}=\mathrm{O}), 1630(\mathrm{C}=\mathrm{N}) .{ }^{1} \mathrm{H} \mathrm{NMR}\left(\mathrm{CDCl}_{3}, \delta \mathrm{ppm}\right): 6.43-7.90(\mathrm{~m}, 13 \mathrm{H}, \mathrm{Ar}-\mathrm{H}), 4.32\left(\mathrm{t}, 2 \mathrm{H}, \mathrm{CH}_{2}\right)$, $3.35(\mathrm{t}, 1 \mathrm{H}, \mathrm{NH}), 2.21(\mathrm{t}, 1 \mathrm{H}, \mathrm{NH})$. Anal. calcd. for $\mathrm{C}_{21} \mathrm{H}_{17} \mathrm{ClN}_{4} \mathrm{O}: \mathrm{C}, 66.93 ; \mathrm{H}, 4.55 ; \mathrm{N}$, 14.87. Found: C, 67.32; H, 4.51; N, 14.43 .

\section{Molecular docking}

The training sets of ligand molecules (Figure 3) were generated through conformational search module and further implementation has been done by Hyperchem Professional 7.0. The fragments were identified on the basis of "Lipinski's Rule of Five" and may therefore represent suitable starting point for evolution of good quality lead compounds. 10 novel ligands were developed for the inhibitory site in DNA gyrase proteins (Figure 4). All the molecular modeling studies were carried out on an Intel Pentium $1.6 \mathrm{GHz}$ processor, 512 MB memory with Windows XP operating system using AutoDockTools (ADT) v 1.5.4 and AutoDock v 4.2. The target sequences of DNA gyrase-II of antimicrobial activity proteins were incurred from UniProt/Swiss-Prot protein knowledgebase (P0A0K8). To run AutoDock to search autogrid by creating Grid maps of different grid points for keeping ligands to cover binding pockets based on active site amino acids within the selected protein. Polar hydrogen was added to the ligand moieties along with Gasteiger-type were assigned and the nonpolar hydrogens were merged with the carbons and the internal degrees of freedom and torsions were set. A set of Lamarckian genetic algorithm was substantially used for molecular docking simulations. The parameters such as population size of 150 , the mutation rate of 0.02 and crossover rate of 0.8 were fixed accordingly. Secondly, the Simulations were performed up to 2.5 million energy and the evaluations were maximum at 27000 generations. Each simulation was carried about 10 times which ultimately yielded 10 docked conformations. From this, the lowest energy conformations were regarded as the best binding conformations.

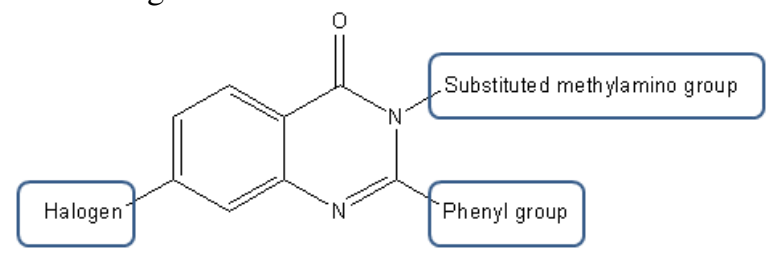

Figure 3. Quinazolinone ligand molecule

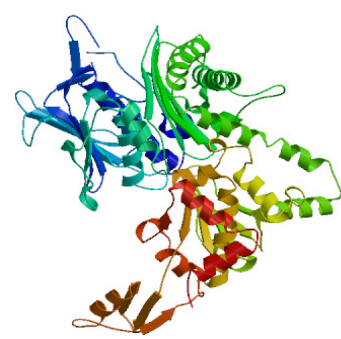

Figure 4. DNA gyrase protein

\section{Preclinical evaluation}

The drug likeness, drug score and theoretical toxicity risks (mutagenic, tumorigenic, irritant and reproductive effects) were also calculated using web-based application for Organic Chemistry Portal, Osiris Property Explorer (http://www.organic-chemistry.org) ${ }^{26}$. 


\section{Antibacterial activity}

Compounds (4a-4j) were assayed for their antibacterial activity against Staphylococcus aureus (MTCC 96) and Escherichia coli (MTCC 723) by cup plate method. Muller Hinton agar plates $\left(37{ }^{\circ} \mathrm{C}, 24 \mathrm{~h}\right)$ were used for cultivation of bacteria. The paper disc diffusion method was carried out in sterilized (autoclaved at $120{ }^{\circ} \mathrm{C}$ for $1 \mathrm{~h}$ ) petri dish. Discs impregnated with $100 \mu \mathrm{g} /$ disc test samples were placed on the surface of agar plates already inoculated with pathogenic bacteria. The plates were incubated at $37{ }^{\circ} \mathrm{C}$ and examined after $24 \mathrm{~h}$ for zone of inhibition. Ampicillin was used as a standard. An additional control disc with an equivalent amount of solvent DMSO was also used in the assay. Further, their minimum inhibitory concentration values against these microorganisms were determined by serial dilution method $^{27}$.

\section{Results and Discussion}

Analytical data, FTIR and ${ }^{1} \mathrm{H}$ NMR confirmed the structure of the new compounds. All compounds shown IR absorption bands at about $3380 \mathrm{~cm}^{-1}$ for $\mathrm{NH}$ group, $2880 \mathrm{~cm}^{-1}$ for $\mathrm{CH}_{2}$ and $1670 \mathrm{~cm}^{-1}$ due to $\mathrm{C}=\mathrm{O}$ stretching vibrations. Also ${ }^{1} \mathrm{H}$ NMR spectra of title compounds $(\mathbf{4 a}-\mathbf{4 j})$ showed the presence of methylene group and the aromatic hydrogens all appeared at the expected shifts.

\section{Molecular docking}

The docking study showed that all the compounds interacted with DNA gyrase protein. Compound (4b) interacted with a binding energy of $-5.99 \mathrm{kcal} / \mathrm{mol}$ with help of 3 hydrogen bonds. Compound (4c) interacted via 3 hydrogen bonds with a binding energy of -5.99 $\mathrm{kCal} / \mathrm{mol}$. Compound (4e) had a binding energy of $-6.53 \mathrm{kcal} / \mathrm{mol}$ and it interacted by 2 hydrogen bonds. All other compounds had less binding energy. Overall, compound (4b) interacted the DNA gyrase with 3 hydrogen bonds and had a good binding energy also. The docking result is shown in Table 1.

Table 1. Binding scores and amino acid interactions of the docked compounds on the active site of DNA gyrase

\begin{tabular}{cccc}
\hline Compd. & Binding energy, kcal/mol & No. of hydrogen bonds & Amino acids \\
\hline $\mathbf{4 a}$ & -5.84 & 1 & GLY85 \\
$\mathbf{4 b}$ & -5.99 & 3 & ARG144 \\
$\mathbf{4} \mathbf{c}$ & -5.99 & 1 & LEU162 \\
$\mathbf{4 d}$ & -4.53 & 2 & LEU162 \\
$\mathbf{4 e}$ & -6.53 & 2 & ARG144, ASN145 \\
$\mathbf{4 f}$ & -5.66 & 1 & GLU164 \\
$\mathbf{4 g}$ & -5.71 & 2 & ARG144 \\
$\mathbf{4 h}$ & -0.50 & 1 & GLU230 \\
$\mathbf{4 i}$ & -4.08 & 3 & ARG144, GLY85 \\
$\mathbf{4 j}$ & -5.84 & 1 & GLU164 \\
\hline
\end{tabular}

\section{Preclinical evaluation}

The toxicity assessment of all the final synthesized compounds depicted in Table 2 shows that all the compounds were free from all type of toxicity issues mentioned in the software package. Table 2 also explains that the presence of lipophilic halogen or electron donating groups in the phenyl nucleus of titled derivatives showed topological polar surface area 
(TPSA) below 51 in compounds (4a-c) and (4g). The low score of TPSA suggests that these molecules preferentially act as hydrophobic in nature and can easily be transported through the blood brain barrier. Drug score of compound (4b) and (4d) is 0.84 and 0.82 respectively which means these have good drug like properties.

Table 2. Toxicity prediction and drug score of synthesized compounds

\begin{tabular}{cccccccc}
\hline Compd. & Mutagenic & Tumerogenic & Irritant & $\begin{array}{c}\text { Reproductive } \\
\text { effect }\end{array}$ & TPSA & $\begin{array}{c}\text { Drug } \\
\text { likeness }\end{array}$ & $\begin{array}{c}\text { Drug } \\
\text { score }\end{array}$ \\
\hline $\mathbf{4 a}$ & NO & NO & NO & NO & 47.94 & 5.82 & 0.76 \\
$\mathbf{4 b}$ & NO & NO & NO & NO & 47.94 & 5.24 & 0.84 \\
$\mathbf{4 c}$ & NO & NO & NO & NO & 47.94 & 4.17 & 0.7 \\
$\mathbf{4 d}$ & NO & NO & NO & NO & 76.96 & 5.02 & 0.82 \\
$\mathbf{4 e}$ & NO & NO & NO & NO & 56.73 & 4.97 & 0.61 \\
$\mathbf{4 f}$ & NO & NO & NO & NO & 76.96 & 4.83 & 0.62 \\
$\mathbf{4 g}$ & NO & NO & NO & NO & 47.94 & 1.98 & 0.37 \\
$\mathbf{4 h}$ & NO & NO & NO & NO & 65.96 & 3.51 & 0.57 \\
$\mathbf{4 i}$ & NO & NO & NO & NO & 102.5 & -2.05 & 0.3 \\
$\mathbf{4 j}$ & NO & NO & NO & NO & 56.73 & 3.38 & 0.58 \\
\hline
\end{tabular}

Antibacterial activity

The antibacterial activity for the synthesized compounds $(\mathbf{4} \mathbf{a}-\mathbf{4} \mathbf{j})$ showed that almost all compounds activity (Table 3 ). Compound (4c) showed comparable antibacterial activity when compared to the standard drug, ampicillin. The MIC value for compound (4c) was found to be $50 \mu \mathrm{g} / \mathrm{mL}$ for both $S$ aureus as well as $E$ coli.

Table 3. Antimicrobial activity of the synthesized compounds

\begin{tabular}{ccccc}
\hline \multirow{2}{*}{ Zone of inhibition (mm) and minimum inhibitory concentration, $\mu \mathrm{g} / \mathrm{mL}$} \\
\hline \multirow{2}{*}{ Compound } & \multicolumn{3}{c}{ S aureus } & \multicolumn{3}{c}{ E coli } \\
\cline { 2 - 5 } & Zone of inhibition & MIC & Zone of inhibition & MIC \\
\hline $\mathbf{4 a}$ & 17 & 100 & 9 & 100 \\
$\mathbf{4 b}$ & 21 & 25 & 11 & 50 \\
$\mathbf{4 c}$ & 20 & 50 & 11 & 50 \\
$\mathbf{4 d}$ & 13 & 75 & 8 & 50 \\
$\mathbf{4}$ & 15 & 50 & 7 & 75 \\
$\mathbf{4 f}$ & 16 & 100 & 8 & 50 \\
$\mathbf{4 g}$ & 14 & 75 & 7 & 100 \\
$\mathbf{4 h}$ & 7 & 100 & 8 & 50 \\
$\mathbf{4 i}$ & 15 & 100 & 9 & 75 \\
$\mathbf{4 j}$ & 12 & 100 & 7 & 50 \\
Ampicillin & 22 & 8 & 12 & 12 \\
\hline
\end{tabular}

\section{Conclusion}

Among the synthesized quinazolinone derivatives under investigation, compound (4b), (4c) and (4e) showed good docking score upon the enzyme DNA gyrase. Toxicity prediction by software package showed that none of the synthetic compounds had any kind of toxicity. It also predicted that compounds (4a-c) and (4g) had good chances of penetrating the blood brain barrier. It also showed that compound (4b) and (4d) had good drug score. The antibacterial activity showed that compound (4b) has comparable activity as that of ampicillin. 
Moreover, it was found that substitution of aliphatic amines in the quinazolinone ring at position 3 increases antibacterial activity. Incorporation of alicyclic amine has moderate activity. It was found that aromatic amine substitution decreased activity. A detailed comparison of the docking study, toxicity study, drug likeliness property and antibacterial activity, it was concluded that the compound (4b) overall showed better properties as a drug like candidate. However search of more potent derivate is underway in our research.

\section{References}

1. Alagarsamy V, Rupeshkumar M, Kavitha K and Meena S, Shankar D, Siddiqui A A and Rajesh R, Eur J Med Chem., 2008, 43(11), 2331-2337;

DOI:10.1016/j.ejmech.2007.10.001

2. Tyagi R, Goel B, Srivastava V K and Kumar A, Indian J Pharm Sci., 1998, 60, 283-286.

3. Alagarsamy V, Muthukumar V, Pavalarani N, Asanthanathan P V and Revathi R, Biol Pharm Bull., 2003, 26(4), 557-559; DOI:10.1248/bpb.26.557

4. Kumar A, Sharma S, Archana, Kiran B, Shipra S and Panwar H, Tripti Singh and Srivastava V K, Bioorg Med Chem., 2003, 11(23), 5293-5299; DOI:10.1016/S09680896(03)00501-7

5. Yadav M R, Shirude S T, Parmar A, Balaraman R and Giridhar R, Chem Heterocycl Compd., 2006, 42(8), 1038-1045; DOI:10.1007/s10593-006-0201-4

6. Varsha J, Pradeep M, Sushil K and Stables J P, Eur J Med Chem., 2008, 43(1), 135141; DOI:10.1016/j.ejmech.2007.02.004

7. Bedi P M S, Kumar V and Mahajan M P, Bioorg Med Chem Lett., 2004, 14(20), 5211-5213; DOI:10.1016/j.bmcl.2004.07.065

8. Jantová S, Stankovský Š and Špirková K, Biologia Bratislava., 2004, 59(6), 741-752.

9. Tran T P, Ellsworth E L, Stier M A, Domagala J M, Showalter H D H and Gracheck S J, Martin A Shapiro, Themis E Joannides and Rajeshwar Singh, Bioorg Med Chem Lett., 2004, 14(17), 4405-4409; DOI:10.1016/j.bmcl.2004.06.063

10. Tale R H, Rodge A H, Keche A P, Hatnapure G D, Padole P R, Gaikwad G S and Turkar S S, J Chem Pharm Res., 2011, 3(2), 496-505.

11. Boehm H J, Boehringer M, Bur D, Gmuender H, Huber W, Klaus W, Kostrewa D, Kuehne H, Luebbers T, Meunier-Keller N, et al., J Med Chem., 2000; 43, 2664-2674.

12. Huband M D, Cohen M A, Zurack M, Hanna D L, Skerlos L A, Mark C S, Glenn W G, Jeffrey W G, Edmund E, Michael A S and Stephen J G, Antimicrob Agents Chemother., 2007, 51(1), 1191-1201; DOI:10.1128/AAC.01321-06

13. Tran T P, Ellsworth E L, Sanchez J P, Watson B M, Stier M A, Hollis Showalter H D, John M D, Martin A S, Themis J E, Stephen J G, Dai Q N, Paul B, Judy Y, Anurag S, Chan H, Saeed R, Xiujuan W and Rajeshwar S, Bioorg Med Chem Lett., 2007, 17(5), 1312-1320; DOI:10.1016/j.bmcl.2006.12.005

14. Wang J C, Untangling the double helix: DNA Entanglement and the Action of the DNA Topoisomerases. Cold Spring Harbor, NY 2009.

15. Eakin A E, Green O, Hales N, Walkup G K, Bist S and Singh A, et al. Antimicrob Agents Chemother., 2012, 56(3), 1240-1246; DOI:10.1128/AAC.05485-11

16. Boyapati S, Kulandaivelu U, Sangu S and Vanga M R, Arch Pharm (Weinheim)., 2010, 43, 570-576.

17. Said M M and Hussein M M M, Bull Fac Pharm Cairo Univ., 1994, 32, 341-347.

18. Mishra P, Panneerselvam P and Jain S, J Indian Chem Soc., 1995, 72, 559-560.

19. Oza H B, Joshi D G and Parekh H H, Heterocycl Commun., 1997, 3, 239-244. 
20. Kumar P, Nath C, Bhargava K P and Shanker K, Pharmazie, 1982, 37, 802-804.

21. Roubinek F, Vavrina J and Budesinsky Z, Collect Czec Chem Commun., 1982, 47, 630-635; DOI:10.2478/acph-2013-0006

22. Zhixiong G Y, Bakshi R K, Chen M, Rohrer S P, Feighner S D and Pong S S, Andrew D H, Allan B, Elizabeth T B, Louis L, Rupa M P, Wanda W S C, James M S, Roy G S, Arthur A P and Ravi P N, Bioorg Med Chem Lett., 2000, 10(1-3), 5-8; DOI:10.1016/S0960-894X(99)00584-3

23. Varma R S, Prakash R and Prasad C R, J Chem Soc Pak., 1986, 8, 117-123.

24. De Souza M V N, Pais K C, Kaiser C R, Peralta M A, Ferreira M L and Lourenço M C S, Bioorg Med Chem., 2009, 17(4), 1474-1480; DOI:10.1016/j.bmc.2009.01.013

25. Alagarsamy V, Murugesan S, Dhanabal K, Murugan M and De Clercq E, Indian J Pharm Sci., 2007, 69, 304-307.

26. Barry A L, The antimicrobial susceptibility test: Principles and Practice, ELBS: London, 1999, 180.

27. Dastidar S G, Chaudhary A, Annadurai S, Roy S, Mookherjee M and Chakrabarty A N, J Chemother., 1995, 7(3), 201-206; DOI:10.1179/joc.1995.7.3.201 